\title{
In Vivo Interaction of Synthetic Acylated Apopeptides with High Density Lipoproteins in Rat
}

\author{
Gabriel Ponsin, James T. Sparrow, Antonio M. Gotto, Jr., and Henry J. Pownall \\ Department of Medicine, Baylor College of Medicine and The Methodist Hospital, 6565 Fannin, Houston, Texas 77030
}

\begin{abstract}
The metabolism of synthetic peptide analogues of high density lipoprotein (HDL) apoproteins has been studied in the rat. These compounds are 15-amino acid lipid associating peptides (LAPs) bearing acyl chains of various lengths (0-16 carbon units). After injection of each ${ }^{125} \mathrm{I}-\mathrm{LAP}$, the serum decay curves suggested a two-compartment process with a clearance rate decreasing when the acyl chain lengths increased. The similarity between the apparent half-life of $\mathrm{C}_{16}$-LAP and that of apoprotein A-I as well as the chromatographic analysis of rat serum were consistent with a partitioning of the LAPs between HDL and the aqueous phase. This was strongly dependent upon the acyl chain length of the LAPs. The distribution volumes of the ${ }^{125}$ I-LAPs in organs were measured $10 \mathrm{~min}$ after injection. The results were analyzed using a model explicitly predicting the organ distribution volumes of HDL and the equilibrium constant $\left(K_{\text {eq }}\right)$ of the binding of each LAP to HDL. HDL distributed significantly in the adrenals (250 $\mu \mathrm{l} / \mathrm{g})$, liver $(80 \mu \mathrm{l} / \mathrm{g})$, and ovaries $(55 \mu \mathrm{l} / \mathrm{g})$, but not in the kidneys. This suggests that the binding of HDL apoproteins to kidneys, reported by others, was due to the uptake of free apoproteins. The $K_{\text {eqs }}$ exhibited a log-linear relationship with respect to the acyl chain length of the LAPs. Each carbon unit added to the acyl chain decreased the free energy of association by a constant value $\left(0.3 \mathrm{kcal} \mathrm{mol}^{-1}\right)$. This clearly showed a strict hydrophobic effect similar to that previously observed in vitro.
\end{abstract}

\section{Introduction}

The plasma lipoproteins are water-soluble complexes that transport lipids in blood (1-3). They have been defined according to their densities as the high density lipoproteins (HDL), low, intermediate, and very low density lipoproteins, as well as the chylomicrons. The lipoproteins are composed of a central core of neutral lipids surrounded by a monomolecular layer of polar lipids and specific apoproteins $(4,5)$. Most of the polar components, including apoproteins, spontaneously exchange among lipoproteins. The mechanism of transfer of apoproteins probably involves transport of molecular species through the aqueous phase (6-9). Consistent with this view, a pool of free apoprotein

Portions of this work were presented at the American Society of Clinical Investigation, Washington, DC, 1984.

Dr. Ponsin is a Robert A. Welch Foundation Postdoctoral Fellow.

Received for publication 9 March 1984 and in revised form 9 September 1985.

J. Clin. Invest.

(c) The American Society for Clinical Investigation, Inc.

0021-9738/86/02/0559/09 \$1.00

Volume 77, February 1986, 559-567
AI (apo A-I), ${ }^{1}$ distinct from HDL, has been identified in human plasma (10). Moreover, studies of apo A-I metabolism in rats led to the assumption of the existence of free apoproteins (11). This implies that the metabolism of a given apoprotein is not necessarily representative of that of the lipoprotein that transports it. Therefore, the partitioning of apoproteins between lipoproteins and the aqueous phase must be considered when studying lipoprotein metabolism. This concept of partitioning has been extensively studied based on the amphipathic helical theory of lipid-binding of apoproteins proposed by Segrest et al. (12). Hypothetically, when an apoprotein assumes a helical structure, the polar and the hydrophobic residues appear on opposite sides of the helix. This arrangement permits the nonpolar face of the helix to penetrate the lipid matrix and the polar face to remain in contact with the aqueous phase. This theory has now been supported by numerous reports involving natural as well as synthetic model apopeptides (13-20). These studies led to the formulation of a number of properties of native or synthetic lipid associating apoproteins, which are mainly: $(a)$ The ability to form an amphipathic $\alpha$-helix; (b) a high hydrophobicity; (c) a minimal length. Although different, these criteria are not independent; therefore, they cannot be clearly distinguished with purely peptidic molecules. To avoid this difficulty, we synthesized a family of acylated peptides having a hydrophobic content that increases with the acyl chain length, whereas the $\alpha$-helical potential and the length of the peptide remain constant. We have recently shown that these peptides bind to HDL, in vitro (21). The dependence of this binding upon the acyl chain length of the peptide reflected a quantitative expression of the hydrophobic effect. We now report the in vivo binding properties and the metabolic fate of HDL containing these acylated peptides. The data have been fitted to a model that specifically includes the partitioning of these peptides between HDL and the aqueous phase.

\section{Methods}

Materials. Female Sprague-Dawley rats (180-200 g) were obtained from Texas Animal Specialties, Houston, TX. ${ }^{125} \mathrm{I}-\mathrm{Na}$ and ${ }^{131} \mathrm{I}-\mathrm{Na}$ were purchased from Amersham Corp., Arlington Heights, IL. Fatty acid free bovine serum albumin was purchased from Sigma Chemical Co., St. Louis, MO. 1-Palmitoyl-2-oleoyl-sn-glycero-3-phosphorycholine(POPC) was obtained from Avanti Polar Lipids, Inc., Birmingham, AL. Rat apo A-I as well as human apo A-I and apo A-II were purified as previously described (20).

1. Abbreviations used in this paper: $\mathrm{ADV}(\mathrm{s})$, apparent distribution volume(s); apo, apoprotein; BDV, bound distribution volume; BS/FS, ratio of HDL-bound peptide to free peptide in serum; $\mathrm{C}_{n}$-LAP(s), lipid associating peptide(s) bearing an acyl chain of $n$ carbons; FDV, free distribution volume; LAP(s), lipid associating peptide(s); NSDV(s), nonspecific distribution volume(s); POPC, 1-palmitoyl-2-oleoyl-sn-glycero-3-phosphorycholine; $[\mathrm{P}]$, molar concentration of the surface lipids of $\mathrm{HDL}$; [W], molar concentration of water. 
The amino acid sequence of the acylated lipid associating peptides (LAPs) is that of the 15-amino-acid COOH-terminal fragment of the previously studied peptide LAP-20 (19). A saturated acyl chain of various numbers of carbons $(0<n<16)$ was covalently bound to the $\mathrm{NH}_{2}$ terminal serine through a peptide bond as previously described (21). Thus, the acylated LAPs had the structure: $\mathbf{R}_{\mathbf{n}}$-SerSerLeuLysGluTyrTrpSerSerLeuLysGluSerPheSer. The nomenclature adopted here is of the form $\mathrm{C}_{n}$-LAP, where $n$ is the number of carbons in the acyl chain of the LAP.

Buffers. All in vitro experiments were carried out using a buffer containing $150 \mathrm{mM} \mathrm{NaCl}, 0.01 \mathrm{M}$ EDTA, $0.01 \mathrm{M}$ sodium azide, and 10 $\mathrm{mM}$ Tris $\cdot \mathrm{HCl}, \mathrm{pH}$ 7.4. The samples were injected into rats in a phosphate-buffered saline (PBS) containing $0.12 \mathrm{M} \mathrm{NaCl}$ and $0.03 \mathrm{M}$ phosphate, $\mathrm{pH}$ 7.4.

Peptide labeling. The LAPs bearing a saturated acyl chain of $n$ carbons ( $C_{n}$-LAPs) were radioiodinated using the chloramine $T$ method (22). The radioiodinated peptides were separated from residual free iodine by gel filtration. Usually all radioactive preparations were used within $2 \mathrm{~d}$ after iodination.

Preparation of reassembled HDL (R-HDL). POPC and apo A-I (100: 1 , molar ratio) were mixed with traces of each ${ }^{125}$ I-LAP in the presence of sodium cholate, at room temperature. The detergent was then separated from the lipid-protein complex by gel filtration on a desalting column of Biorad P4 (Bio-Rad Laboratories, Richmond, CA) (23). This procedure also separated the fraction of ${ }^{125}$ I-LAP that was not associated with the POPC/apo A-I complex (21).

Serum clearance measurements. To avoid any significant formation of ${ }^{125}$ I-labeled thyroid hormones during serum decay experiments, we injected the rats intraperitoneally with $1 \mathrm{ml}$ of potassium iodide $(1 \mathrm{mg} /$ $\mathrm{ml}) 1 \mathrm{~d}$ before and at the time of sample injection. Catheters were introduced into the femoral veins of rats on both sides under ether anesthesia. One catheterized vein was used for injecting the sample and the other for periodic blood sampling. Between samplings, a solution of $0.9 \%$ $\mathrm{NaCl}$ was infused into the catheter at a rate of $1 \mathrm{ml} / \mathrm{h}$. The samples to be injected consisted of ${ }^{125}$ I- $C_{n}$ LAPs $\left(5-10 \times 10^{6} \mathrm{cpm}\right)$ either as R-HDL components or as free molecules in PBS. These two ways of preparing the samples gave indistinguishable results. The sera obtained from periodic blood sampling (100-200 $\mu$ l) were counted for ${ }^{125}$ I radioactivity. The experiments were continued until the total serum radioactivity was $<1 \%$ of the injected radioactivity. The serum volume was considered at $4 \%$ of body weight.

In vivo binding of ${ }^{125}$ I-LAPs to organs. Rats under ether anesthesia were injected via a femoral vein with each ${ }^{125}$ I-LAP either as a component of R-HDL, as free molecules in PBS, or in rat serum after overnight equilibration. Injection by these three methods gave indistinguishable results and therefore all were included in subsequent calculations. The rats were exsanguinated via the abdominal aorta, 5-7 min after injection. Then, the liver, kidneys, adrenals, ovaries, spleen, abdominal muscle, heart, lungs, and stomach were immediately removed (normally within $10 \mathrm{~min}$ after injection). After careful cleaning, each organ was weighed and counted for ${ }^{125}$ I radioactivity. Entire organs were counted with the exception of muscle and liver, for which only pieces were counted. In the latter case, several pieces from different parts of the liver were mixed before counting. The results were expressed in terms of a volume of serum having the same radioactivity; thus, the apparent distribution volumes (ADVs) had the units of $\mu \mathrm{l} / \mathrm{g}$ of organ. The nonspecific association of radioactivity with the organs, due to the presence of residual serum after removal, was estimated by measuring the organ distribution volumes of albumin (24). This was determined after injection of ${ }^{125} \mathrm{I}$-albumin to specific rats or after injection of ${ }^{131} \mathrm{I}$-albumin together with ${ }^{125} \mathrm{I}$-LAPs into the same rats. Both procedures gave equivalent results. For convenience, the organ distribution volumes of albumin will be referred to as the nonspecific distribution volumes (NSDVs).

Modeling of LAP metabolism. The LAP ADVs were fitted to a model that has been fully developed elsewhere (25). This model can be summarized as follows: each LAP distributes in serum between HDL and the aqueous phase. This partitioning is assumed to change with respect to the acyl chain length of the LAP. The model expressly relates the partitioning of the LAPs in serum to their organ ADVs according to:

$\mathrm{ADV}_{i j}=\left(\mathrm{FDV}_{i}+\mathrm{NSDV}_{i}\right)+\left(\mathrm{BDV}_{i}-\mathrm{FDV}_{i}\right) \frac{\mathrm{BS}_{j}}{\mathrm{TS}_{j}}$

where $\mathrm{ADV}_{i j}$ is the $\mathrm{ADV}$ of the $j^{\text {th }} \mathrm{LAP}$ in the $i^{\text {th }}$ organ. $\mathrm{FDV}_{i}, \mathrm{BDV}_{i}$ are the distribution volumes in the $i^{\text {th }}$ organ of the free LAP and of the HDL-bound LAP, respectively. $\mathrm{NSDV}_{i}$ is the nonspecific distribution volume in the $i^{\text {th }}$ organ. $\mathrm{BS}_{j} / \mathrm{TS}_{j}$ represents the fraction of the $j^{\text {th }}$ LAP bound to $\mathrm{HDL}\left(\mathrm{BS}_{j}\right)$ relative to total LAP $\left(\mathrm{TS}_{j}\right)$ in serum. Equation 1 can be rewritten as:

$\Delta_{i} \frac{\mathrm{BS}_{j}}{\mathrm{TS}_{j}}=\mathrm{ADV}_{i o}-\mathrm{ADV}_{i j}$,

where $\Delta_{\mathrm{i}}=\mathrm{FDV}_{i}-\mathrm{BDV}_{i}$ and $\mathrm{ADV}_{i o}$ is the $\mathrm{ADV}$ in the $i^{\text {th }}$ organ of a theoretical LAP whose binding to HDL is nil.

Application of equation 2 to two different organs $(i=a$ and $i=b)$ leads to:

$\mathrm{ADV}_{a j}=\frac{\Delta_{a}}{\Delta_{b}} \mathrm{ADV}_{b j}+\mathrm{ADV}_{a o}-\frac{\Delta_{a}}{\Delta_{b}} \mathrm{ADV}_{b o}$

which predicts a linear relationship between the ADV of any organ $a$ and the ADV of any organ $b$ when $j$ varies. This relationship permits the calculation of $\mathrm{ADV}_{o}$ and $\Delta$ in any organ, after estimation of these two parameters in only one organ taken for reference. It then becomes possible to estimate $\mathrm{FDV}_{i}, \mathrm{BDV}_{i}$, and $\mathrm{BS}_{j} / \mathrm{TS}_{j}$ from equations 1 and 2.

In vitro binding to tissue membranes. The tissue preparation and binding assays were based on those previously described (26). Fresh adrenals, ovaries, and livers from female rats were rinsed in chilled saline and homogenized. The homogenates were centrifuged at $1,000 \mathrm{~g}$ for 15 $\min$. The resulting supernatant will be referred to as crude tissue membranes in this report.

Rat HDL were prepared by sequential ultracentrifugation in the 1.09 to 1.21 density range (27). ${ }^{125} \mathrm{I}_{-} \mathrm{C}_{16}$ - $\mathrm{LAP}$ labeled HDL were incubated for $30 \mathrm{~min}$ at $37^{\circ} \mathrm{C}$ with crude tissue membranes in the presence of various amounts of unlabeled rat HDL. Nonspecific binding was estimated by addition of a 500-fold excess of unlabeled rat HDL. Aliquots of the samples were layered on $2 \mathrm{ml}$ of chilled Tris buffer containing $1 \%$ bovine serum albumin. Bound HDL were separated from free HDL by centrifugation $(30,000 \mathrm{~g}$ for $30 \mathrm{~min})$. The pellets were washed once and counted for ${ }^{125} \mathrm{I}$ radioactivity. Further details are provided in the legend to Fig. 4.

\section{Results}

The serum clearances of the acyl peptides were measured after a bolus intravenous injection of each ${ }^{125} \mathrm{I}$-LAP. The clearance rate dramatically decreased when the acyl chain length of the LAP increased (Fig. 1). The decay curves were apparently biexponential, therefore revealing a process involving at least two compartments. After $2 \mathrm{~h}$ and $30 \mathrm{~min}$, the curves reached a pseudo-linearity. The apparent half-life of the long component exhibited a log-linear relationship with respect to the acyl chain length of the LAP. This indicated a decrease in the contribution of the fast component to the apparent half-life of the long component, as the acyl chain length increased. Interestingly, the apparent half-life of the long component of $C_{16}-\mathrm{LAP}$ (513 \pm 12 min) was comparable to that of rat apo A-I reported by others (11), which is consistent with the expected high binding of $\mathrm{C}_{16}$-LAP to HDL. The correlation of the acyl chain lengths of the LAPs with their affinities for HDL was confirmed by gel filtration (Sepharose CL-4B) of the serum of rats killed 30 min after injection. The results obtained with $\mathrm{C}_{0}$-LAP, $\mathrm{C}_{8}$-LAP, and $\mathrm{C}_{16}$-LAP are 


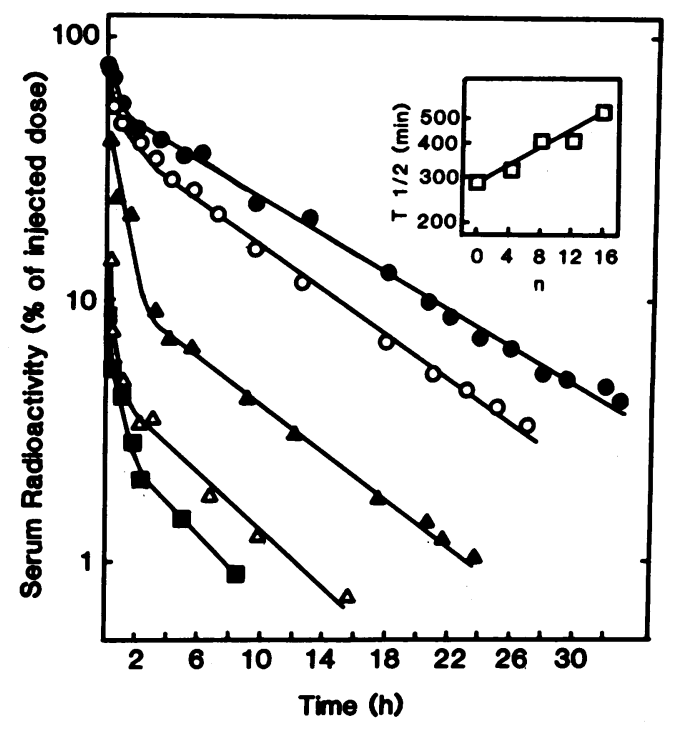

Figure 1. Serum decay curves of ${ }^{125}{ }^{\mathrm{I}}-\mathrm{C}_{\mathrm{n}}$-LAPs. $\mathrm{C}_{0}$-LAP, $\varpi$; $\mathrm{C}_{4}$-LAP, $\Delta$; $\mathrm{C}_{8}$-LAP, $\triangle ; \mathrm{C}_{12}$-LAP, $\mathrm{O}$; and $\mathrm{C}_{16}$-LAP, $\bullet$ were injected into a femoral vein at time 0 . Blood was periodically sampled from the contralateral femoral vein. After $2.5 \mathrm{~h}$ the curves reached an apparent linearity. The inset shows the corresponding apparent half-lives $\left(T_{1 / 2}\right)$ plotted vs. the number of carbon units of the acyl chain of the LAP $(n)$. Each point corresponds to the mean of at least six rats.

shown as examples in Fig. 2. $\mathrm{C}_{0}$-LAP was almost completely recovered as free peptide, whereas $C_{16}$-LAP essentially eluted in the HDL fractions. The elution pattern of $\mathrm{C}_{8}$-LAP was intermediary between those of $\mathrm{C}_{0}$ - and $\mathrm{C}_{16}$-LAP. The binding of ${ }^{125} \mathrm{I}$ -

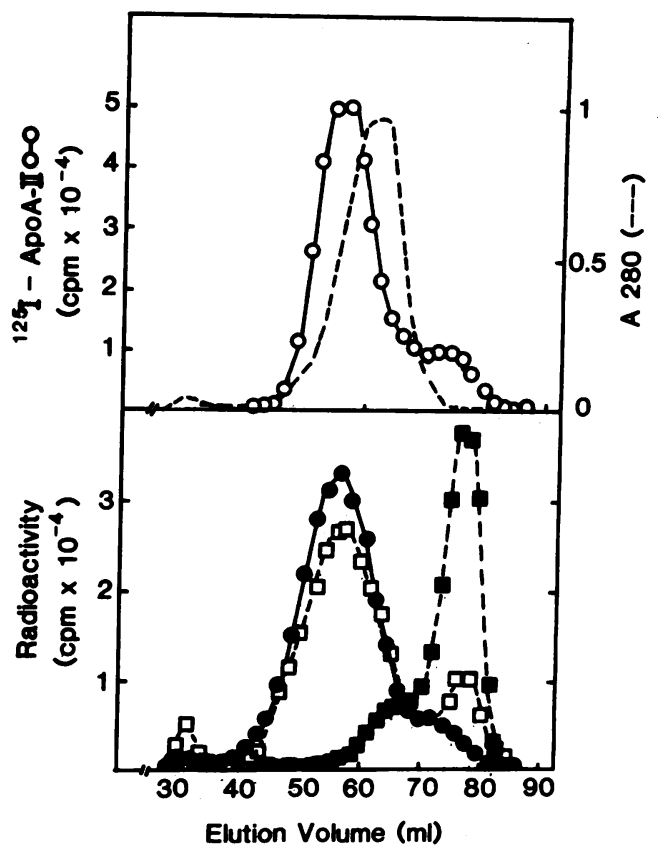

Figure 2. Elution profiles of serum ${ }^{125} \mathrm{I}_{-} \mathrm{C}_{\mathrm{n}}-\mathrm{LAP}$ on a Sepharose CL-4B column, $30 \mathrm{~min}$ after injection. Bottom, elutions of $\mathrm{C}_{0}$-LAP, $\mathbf{m} ; \mathrm{C}_{8}-$ LAP, 口; and $\mathrm{C}_{16}-\mathrm{LAP}, \bullet$. Top, absorbance at $280 \mathrm{nM}$ and the position of HDL peak as judged from the chromatography of rat serum equilibrated with ${ }^{125} \mathrm{I}$-apo A-II. Salt peak had an elution volume of 77-79 ml.
LAPs to organs was measured $1 \mathrm{~d}$ after injection. The level of radioactivity in the organs was much lower after injection of $\mathrm{C}_{0^{-}}$ LAP than after injection of $\mathrm{C}_{16}$-LAP (Fig. 3). This suggested that the total body clearance of the former was much faster than that of the latter. When expressed per gram of organ, the radioactivity of $\mathrm{C}_{0}$-LAP was predominantly associated with kidneys, which suggests that this organ was responsible for the catabolism of the free peptide. The distribution of the $C_{16}$-LAP radioactivity was markedly different. It exhibited a preferential labeling of adrenals, ovaries, liver, and spleen. After $1 \mathrm{~d}$, quantitative analysis of these data could not be done because of the catabolism and/ or excretion, and possible recirculation of radioiodinated compounds. However, qualitatively, the distribution of $\mathrm{C}_{16}$-LAP radioactivity closely resembled that of HDL (24) and therefore remained consistent with a binding of the acyl peptide to HDL. As judged from in vitro experiments, the association of LAP to HDL did not affect the normal behavior of the lipoprotein. ${ }^{125} \mathrm{I}$ $\mathrm{C}_{16}$-LAP labeled HDL were able to bind to crude membranes from either adrenals, ovaries or livers; these were displaced by $>90 \%$ in the presence of unlabeled rat HDL (Fig. 4).

Since the affinities of the LAPs for HDL appeared to be dependent upon their acyl chain length, we measured the in vivo binding of each ${ }^{125}$ I-LAP to organs, 10 min after injection. The results were expressed in terms of ADVs having the unit of microliters per gram of organ. We elected to measure the organassociated radioactivity after $10 \mathrm{~min}$ because it is a time long enough for the ADVs to reach equilibrium (24), and sufficiently short for the radioactivity to be representative of the injected compound. Chromatography of serum on Sephadex G-25 column did not reveal the presence of either free radioactive iodine or ${ }^{125} \mathrm{I}$-tyrosine. Moreover, injection of cold NaI $(150 \mu \mathrm{g} / \mathrm{rat})$ with the samples did not change the ADVs in any of the organs tested. In the first group of experiments, the effects of peptide dose were evaluated. Since the most hydrophobic LAPs have been shown to self-associate (21), we estimated the in vitro degree of self-association of $\mathrm{C}_{16}$-LAP as a function of concentration by measuring the shift of the intrinsic fluorescence of tryptophan as previously described (21). The shifts of $\lambda_{\max }$ were $-11,-10$, -7 , and -1 at $100,10,1$, and $0.1 \mu \mathrm{M}$, respectively. Use of lower concentrations precluded accurate fluorescence measurements. In vivo, when only traces of ${ }^{125} \mathrm{I}-\mathrm{C}_{16}$ - $\mathrm{LAP}$ were injected into rats, the concentration of peptide in serum was too low $(\sim 2.5 \mathrm{nM})$ to permit any significant self-association. Injection of 20 or 400

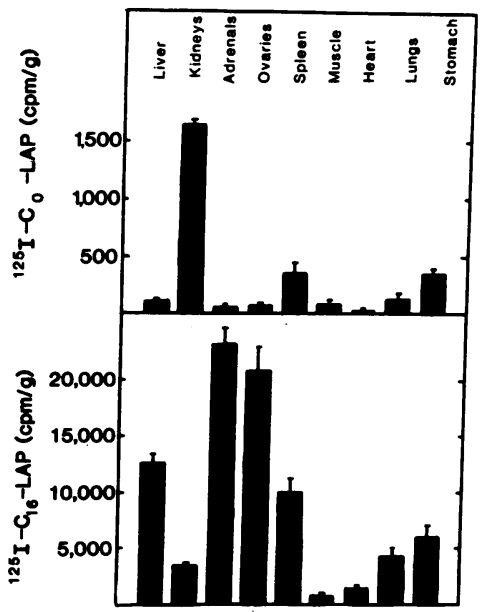

Figure 3. Association of ${ }^{125} \mathrm{I}-\mathrm{C}_{\mathrm{n}}$-LAPs with organs, 1 $\mathrm{d}$ after injection. Rats were bled from abdominal aorta $1 \mathrm{~d}$ after injection of $\mathrm{C}_{0^{-}}$ LAP or $\mathrm{C}_{16}$-LAP. Radioactívity associated with the organs was measured after flushing with $20 \mathrm{ml}$ of saline. Results are expressed as the mean \pm SEM, for six rats, of the radioactivity per gram of organ for $10^{6} \mathrm{cpm}$ injected. 


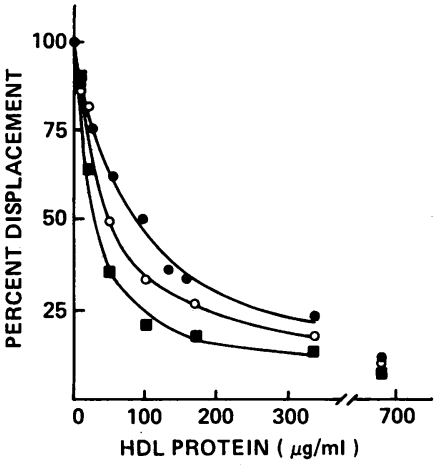

HDL were $<10 \%$ of maximal binding. They were considered as nonspecific binding and were subtracted from all other values.

$\mu \mathrm{g}$ of $\mathrm{C}_{16}$-LAP corresponded to initial peptide concentrations in plasma of $\sim 1$ and $20 \mu \mathrm{M}$, respectively. At these concentrations, $\mathrm{C}_{16}$-LAP might self-associate as judged from the fluorescence measurements mentioned above. In fact, because of the strong association with HDL, the real concentration of unbound peptide in plasma was unknown. Therefore, we estimated the in vivo LAP self-association as follows: serum of rats injected with various amounts of $\mathrm{C}_{16}$-LAP were chromatographed on Sepharose CL-4B. Radioactivity eluting as free peptide was then chromatographed on Sephadex G-25 (Fig. 5). After injection of either traces or $20 \mu \mathrm{g}$ of $\mathrm{C}_{16}$-LAP, the unbound peptide was recovered as monomers. In contrast, after injection of $400 \mu \mathrm{g}, \sim 15 \%$ of $\mathrm{C}_{16}$-LAP eluted as self-associated peptide.

Then, we measured the effect of peptide dose on the tissue ADVs. Representative results obtained with $C_{4}-$ LAPs and $C_{16-}$ LAPs are shown as examples in Table I. The organ ADVs were

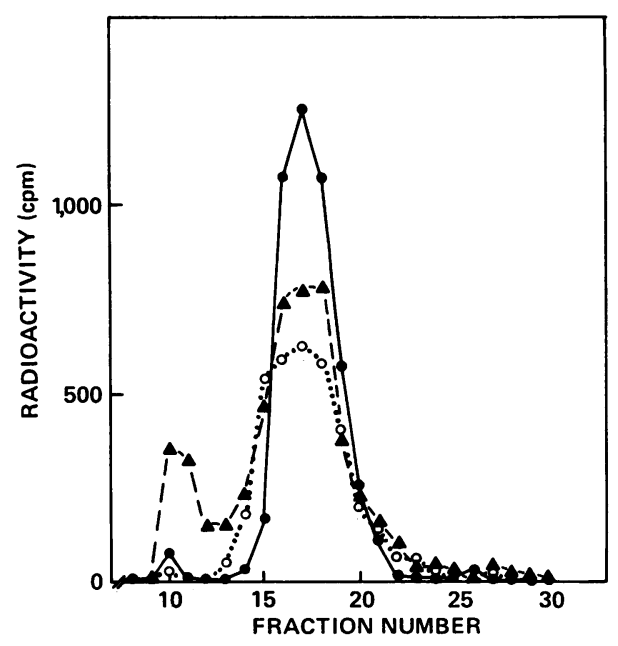

Figure 5. Physical state of ${ }^{125} \mathrm{I}-\mathrm{C}_{16}$-LAP in rat serum as a function of concentration. Rats were sacrificed $10 \mathrm{~min}$ after injection of traces of labeled peptide together with various amounts of unlabeled $\mathrm{C}_{16}$-LAP. HDL-bound and free peptides were separated on a small column of Sepharose CL-4B $(10 \mathrm{ml})$. The fractions corresponding to unbound peptide were then chromatographed on a Sephadex G 25 column (10 $\mathrm{ml})$. The elution profiles correspond to rats injected with $0(\bullet), 20 \mu \mathrm{g}$ (o), or $400 \mu \mathrm{g}(\Delta)$ of $\mathrm{C}_{16}$-LAP. Monomeric LAP eluted at fractions 14 20 , whereas self-associated peptide eluted with or close to the void volume (fraction 9-12) as judged from chromatography of $10 \mu \mathrm{M} \mathrm{C}_{0}$ LAP and $10 \mu \mathrm{M} \mathrm{C}_{16}$-LAP, respectively. independent of the amount of peptide injected and were therefore averaged for subsequent calculations. Table II shows the distribution volumes obtained with six different LAPs $\left(\mathrm{C}_{0^{-}}, \mathrm{C}_{4^{-}}, \mathrm{C}_{6^{-}}\right.$, $\mathrm{C}_{8^{-}}, \mathrm{C}_{12^{-}}$, and $\mathrm{C}_{16^{-}} \mathrm{LAP}$ ) and with albumin. These data were fitted to the model described in Methods.

The ADVs of the different LAPs in each organ were plotted vs. those of muscle (Fig. 6). Muscle was chosen as a reference because it was found to be the organ presenting the least individual variability (Table II). According to equation 3, these plots are straight lines from which we can estimate $\Delta$ and $A_{D V}$ in each organ, provided we know these two parameters in muscle. The ADV of $\mathrm{C}_{0}$-LAP in muscle was used as a first estimate of $\mathrm{ADV}_{0}$. This was justified on the basis of two considerations: (a) previous in vitro data have shown that the binding of $\mathrm{C}_{0}$-LAP to HDL is extremely low (21); and (b) the results from Fig. 2 showed that $30 \mathrm{~min}$ after injection, the association of $\mathrm{C}_{0}$-LAP with HDL was immeasurably low. Estimation of $\Delta$ in muscle is readily done since HDL do not significantly bind to that organ (bound distribution volume $[\mathrm{BDV}]=0)(24)$. Therefore, in muscle, $\Delta$ is identical to FDV, which is the difference between $\mathrm{ADV}_{0}$ and NSDV previously estimated. The difference $\mathrm{ADV}_{0^{-}}$ ADV was calculated for each LAP and plotted vs. the corresponding $\Delta$. According to equation (2), we obtained a group of six straight lines, going through the origin, whose slopes corresponded to the partition coefficients, BS/TS, of each peptide (Fig. 7). From these, we calculated the ratio of HDL-bound peptide to free peptide in serum, BS/FS. This ratio exhibited a loglinear relationship with respect to the acyl chain length of the LAP (Fig. 8). The extrapolated values were used to obtain a definitive estimate of BS/TS, from which we calculated the final values of $\Delta$ and $A D V_{0}$ in each organ (equation 2). The plot of the experimental ADVs vs. BS/TS for each organ is a straight line whose slope is $-\Delta$ and the $y$ intercept is $\mathrm{ADV}_{0}$ (Fig. 9). From these estimates we used equation 2 to obtain the "calculated values" of $\mathrm{ADV}_{i j}$ and we plotted them vs. the experimental $\mathrm{ADV}_{i j}$ (Fig. 10). The high correlation obtained $(r=0.984)$ clearly showed the overall validity of the model. Finally, we calculated $\mathrm{FDV}_{\mathrm{i}}$ and $\mathrm{BDV}_{\mathrm{i}}$ and verified the accuracy of the model for each individual organ as follows: we observed (Fig. 8) that the partitioning of the LAPs between HDL and the aqueous phase was related to their acyl chain lengths according to an equation of the form: $\mathrm{Ln} \mathrm{BS}_{j} / \mathrm{FS}_{j}=K n_{j}+A$, where $K$ and $A$ are constants and $n_{j}$ is the number of carbon units of the acyl chain of the $j^{\text {th }}$ LAP. As previously discussed (25), this implies that the $\mathrm{ADV}_{i j}$ are related to $n_{j}$ according to:

$\mathrm{ADV}_{i j}=\frac{\mathrm{BDV}_{i} \mathrm{e}^{\mathrm{A}} \mathrm{e}^{K n_{j}}+\mathrm{FDV}_{i}}{\mathrm{e}^{\mathrm{A}} \mathrm{e}^{K n_{j}}+1}+\mathrm{NSDV}_{i}$

This equation predicts a sigmoidal relationship between $\mathrm{ADV}_{i j}$ and $n_{j}$. The fit of the experimental data to the theoretical curves indicated that this prediction was satisfied for each organ (Fig. 11). This validated the estimates of $\mathrm{BDV}_{i}, \mathrm{FDV}_{i}$, and $\mathrm{BS}_{j} / \mathrm{FS}_{j}$, which could be considered as being of physiological relevance. The analysis of $\mathrm{BDV}_{i}$ and $\mathrm{FDV}_{i}$ confirmed the results suggested by the 1-d experiments mentioned above (Fig. 12). The free peptide was nearly homogeneously distributed into all organs with the exception of kidneys, where FDV was clearly higher. In contrast, significant quantities of the HDL-bound peptide were associated with only three organs: adrenals, liver, and ovaries. Although adrenals contained more HDL-bound peptide per gram of tissue, the liver had the largest HDL distribution 
Table I. Effect of the Injected Amount of LAPs on their Organ Distribution Volumes

\begin{tabular}{|c|c|c|c|c|c|c|c|c|c|c|}
\hline & \multirow{2}{*}{$\begin{array}{l}\text { Amount } \\
\text { injected }\end{array}$} & \multicolumn{9}{|c|}{ Organ distribution volumes } \\
\hline & & Liver & Kidneys & Adrenals & Ovaries & Spleen & Muscle & Heart & Lungs & Stomach \\
\hline & $\mu g / r a t$ & $\mu l / g$ & $\mu l / g$ & $\mu l / g$ & $\mu l / g$ & $\mu l / g$ & $\mu l / g$ & $\mu l / g$ & $\mu l / g$ & $\mu l / g$ \\
\hline \multirow[t]{4}{*}{$\mathrm{C}_{4}$-LAP } & Traces & $425 \pm 18$ & $1,141 \pm 8$ & $418 \pm 54$ & $427 \pm 36$ & $342 \pm 84$ & $131 \pm 25$ & $297 \pm 25$ & $447 \pm 13$ & $317 \pm 50$ \\
\hline & 0.9 & $447 \pm 14$ & $1,086 \pm 59$ & $382 \pm 38$ & $447 \pm 32$ & $375 \pm 8$ & $142 \pm 2$ & $314 \pm 16$ & $444 \pm 24$ & $379 \pm 8$ \\
\hline & 9 & $415 \pm 28$ & $1,027 \pm 27$ & $368 \pm 30$ & $421 \pm 16$ & $378 \pm 38$ & $151 \pm 15$ & $296 \pm 19$ & $421 \pm 28$ & $344 \pm 37$ \\
\hline & 90 & $446 \pm 10$ & $928 \pm 31$ & $421 \pm 29$ & $484 \pm 21$ & $363 \pm 17$ & $162 \pm 3$ & $344 \pm 18$ & $473 \pm 23$ & $385 \pm 27$ \\
\hline \multirow[t]{3}{*}{$\mathrm{C}_{16}$-LAP } & Traces & $139 \pm 23$ & $106 \pm 23$ & $319 \pm 30$ & $125 \pm 2$ & $75 \pm 7$ & $19 \pm 3$ & $52 \pm 9$ & $70 \pm 9$ & $16 \pm 3$ \\
\hline & 20 & $145 \pm 5$ & $184 \pm 8$ & $349 \pm 25$ & $146 \pm 36$ & $75 \pm 3$ & $13 \pm 1$ & $46 \pm 6$ & $69 \pm 16$ & $21 \pm 2$ \\
\hline & 400 & $134 \pm 22$ & $187 \pm 20$ & $354 \pm 3$ & $171 \pm 21$ & $73 \pm 4$ & $11 \pm 1$ & $32 \pm 4$ & $64 \pm 11$ & $19 \pm 3$ \\
\hline
\end{tabular}

Traces of ${ }^{125}$ I-LAPs were injected with increasing amounts of the same nonradioactive LAPs. The distribution volumes per gram of organs were determined $10 \mathrm{~min}$ after injection. Values are given as the mean $\pm \mathrm{SEM}$ of four rats.

volume per total organ $(600 \mu \mathrm{l})$. The estimates of $\mathrm{BDV}_{i}$ were very close to the organ distribution volumes observed after injection of rat ${ }^{125} \mathrm{I}$-apo A-I-labeled HDL, with the exception of kidneys. The kidney $\mathrm{BDV}_{i}$ was close to nil, whereas this organ exhibited extensive labeling after injection of rat ${ }^{125} \mathrm{I}$-apo A-Ilabeled HDL (Fig. 12).

The estimates of BS/FS were used to calculate the in vivo equilibrium constants $\left(K_{\text {eq }} s\right)$ for the binding of each LAP to HDL, according to: $K_{e q}=\mathrm{BS} / \mathrm{FS} \times[\mathrm{W}] /[\mathrm{P}]$, where [W] is the molar concentration of water $(55.5 \mathrm{M})$ and $[\mathrm{P}]$ is the molar concentration of the surface lipids of HDL. To a first approximation, the latter is the molar concentration of HDL phospholipids. This was considered to be $0.65 \mathrm{mM}$ according to an average of literature data reviewed by Chapman (28). Since $K_{\text {eq }}$ is proportional to BS/FS, it exhibited a log-linear relationship with respect to the acyl chain length of the LAP. This implied that the contribution of each carbon unit of the acyl chain to the free energy of association $\left(\Delta \mathrm{G}_{\mathrm{a}}\right)$ was the same, since: $\Delta \mathrm{G}_{\mathrm{a}}=-R T \operatorname{Ln} K_{\mathrm{eq}}$, where $R$ is the gas constant and $T$ the absolute temperature. This contribution was $-0.3 \mathrm{kcal} \mathrm{mol}^{-1}$ per carbon unit. The in vivo $K_{\text {eq }} s$ were compared with those previously observed in vitro for the binding of the same LAPs to POPC/apo A-I complex
(21). The two groups of $K_{\mathrm{eq}} \mathrm{s}$ were strictly correlated $(r=0.985$; Fig. 13). For each LAP, the in vivo and in vitro $K_{\text {eq }}$ s were of the same order of magnitude, although the former were approximately three times higher than the latter.

\section{Discussion}

There is a growing body of evidence indicating that apoproteins undergo a partitioning between lipoproteins and the aqueous phase of serum (6-11). This concept leads to two considerations. First, free and lipoprotein-bound apoproteins may have different catabolic sites; one cannot, therefore, assume that the metabolism of an apoprotein is necessarily representative of that of the lipoprotein that transports it. Second, the relative amounts of a given apoprotein catabolized as free monomeric species and as a lipoprotein component may be a function of their partitioning between lipoproteins and the aqueous phase. Therefore, the development of criteria that regulate the equilibrium of apoproteins between lipoproteins and the aqueous phase is important to our overall understanding of lipoprotein metabolism. In this report, we measured the metabolism of a series of model HDL apolipopeptides in the rat. These consisted of a family of acylated

Table II. Organ Distribution Volumes of $C_{n}-L A P s$

\begin{tabular}{|c|c|c|c|c|c|c|c|c|c|}
\hline & \multicolumn{9}{|c|}{ Organ distribution volumes } \\
\hline & Liver & Kidneys & Adrenals & Ovaries & Spleen & Muscle & Heart & Lungs & Stomach \\
\hline & $\mu l / g$ & $\mu l / g$ & $\mu l / g$ & $\mu l / g$ & $\mu l / g$ & $\mu l / g$ & $\mu l / g$ & $\mu l / g$ & $\mu l / g$ \\
\hline $\mathrm{C}_{0}$-LAP (8) & $449 \pm 51$ & $1,172 \pm 125$ & $362 \pm 48$ & $501 \pm 26$ & $388 \pm 62$ & $171 \pm 5$ & $269 \pm 9$ & $443 \pm 34$ & $389 \pm 42$ \\
\hline $\mathrm{C}_{4}$-LAP (20) & $405 \pm 14$ & $1,065 \pm 20$ & $401 \pm 10$ & $441 \pm 22$ & $360 \pm 14$ & $143 \pm 5$ & $293 \pm 7$ & $404 \pm 21$ & $347 \pm 27$ \\
\hline$C_{6}$-LAP (8) & $372 \pm 32$ & $904 \pm 16$ & $322 \pm 17$ & $355 \pm 36$ & $234 \pm 8$ & $111 \pm 16$ & $179 \pm 6$ & $287 \pm 13$ & $230 \pm 15$ \\
\hline$C_{8}$-LAP (12) & $257 \pm 11$ & $310 \pm 19$ & $359 \pm 36$ & $178 \pm 6$ & $109 \pm 3$ & $43 \pm 3$ & $76 \pm 7$ & $104 \pm 5$ & $75 \pm 11$ \\
\hline $\mathrm{C}_{12}$-LAP (8) & $211 \pm 25$ & $182 \pm 12$ & $304 \pm 52$ & $173 \pm 20$ & $107 \pm 11$ & $28 \pm 1$ & $40 \pm 1$ & $77 \pm 1$ & $27 \pm 6$ \\
\hline$C_{16}$-LAP (16) & $139 \pm 6$ & $127 \pm 13$ & $332 \pm 9$ & $138 \pm 5$ & $75 \pm 2$ & $14 \pm 1$ & $47 \pm 2$ & $69 \pm 15$ & $17 \pm 1$ \\
\hline Albumin (24) & $86 \pm 19$ & $73 \pm 10$ & $73 \pm 18$ & $70 \pm 8$ & $66 \pm 6$ & $14 \pm 3$ & $30 \pm 9$ & $51 \pm 18$ & $8 \pm 2$ \\
\hline
\end{tabular}

Each ${ }^{125}$ I-LAP was injected into the femoral vein of rats under ether anesthesia. The distribution volumes per gram of organs were determined 10 min after injection. The nonspecific association of radioactivity with organs, due to the presence of residual serum after removal, was estimated by measuring the tissue distribution volumes of ${ }^{125} \mathrm{I}$-albumin. Values are given as the mean \pm SEM. The numbers of injected rats are given in parenthesis. 


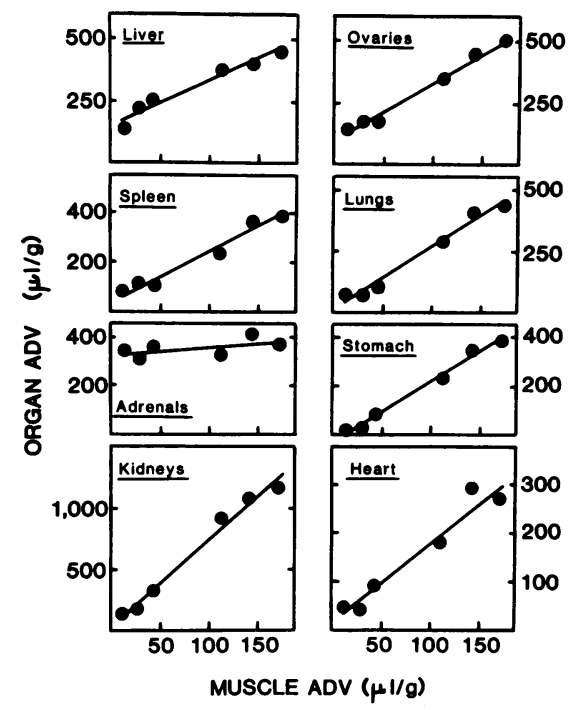

Figure 6. First estimates of the model parameters. The apparent distribution volumes in organs of the $\mathrm{C}_{\mathrm{n}}$-LAPs (ADVs) were plotted vs. those obtained in muscle, according to the model described under Methods (equation 3). From the slopes and the $y$-intercepts of these straight lines, we calculated, respectively, $\Delta$ and $\mathrm{ADV}_{0}$ in each organ. $\Delta$ is the difference between the distribution volumes of the free and HDL bound LAP. ADV $\mathrm{A}_{0}$ is the apparent distribution volume of a theoretical LAP whose affinity for HDL is nil.

LAPs whose acyl chain length varied from 0 to 16 carbon units. Previous studies have shown that these LAPs bound specifically to HDL in vitro (21). This was also true in vivo since we did not observe any binding of the LAPs to lipoprotein other than with HDL. The data were fitted to a model that we previously developed to study HDL metabolism (25). This model takes into account the partitioning of the LAPS between HDL and the aqueous phase. Both HDL and free LAP are assumed to be representative of a single compartment. In fact, because of selfassociation, the free LAP compartment might be, from a theoretical viewpoint, divided into two distinct compartments: the monomeric and the self-associated LAP, respectively. This distinction is not justified on the basis of two considerations: (a) in most experiments, only traces of radioiodinated LAPs were injected, leading to plasma concentrations too low to permit any significant self-association; $(b)$ in a few cases, when larger amounts of LAPs were injected, a limited self-association of only the most hydrophobic LAP was observed. However, this

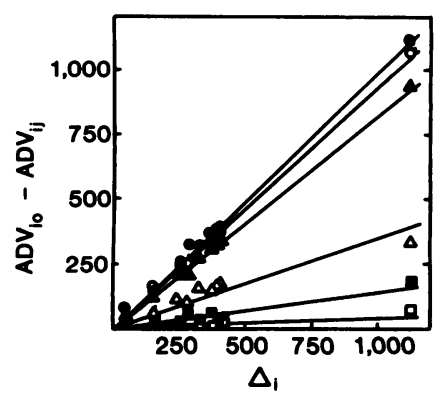

Figure 7. First estimates of the partition coefficients of the $C_{n-}$ LAPs between HDL and the aqueous phase. According to equation 2 of the model described in Methods, the plot of $\mathrm{ADV}_{i o}-\mathrm{ADV}_{i j}$ vs. $\Delta_{i}$ results in a family of straight lines, going through the origin. The slopes correspond to the fraction of $\mathrm{C}_{\mathrm{n}}$-LAP bound to HDL $\left(\mathrm{BS}_{j} /\right.$

$\mathrm{TS}_{j}$ ) obtained with $\mathrm{C}_{0}-\mathrm{LAP}, \square$;

$\mathrm{C}_{4}$-LAP, $₫$; $\mathrm{C}_{6}$-LAP, $\Delta ; \mathrm{C}_{8}$-LAP, $\triangle$; $\mathrm{C}_{12}$-LAP, o; and $\mathrm{C}_{16}$-LAP, $\bullet . \mathrm{ADV}_{i j}$ is the apparent distribution volume of the $j^{\text {th }}$ LAP in the $i^{\text {th }}$ organ. Other parameters are defined in Fig. 5.

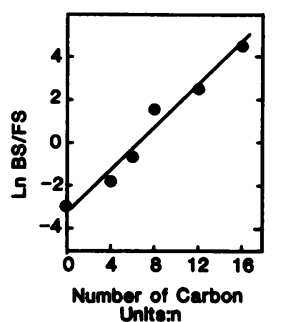

did not change the tissue distribution volumes of the LAPs, showing that a limited LAP self-association does not significantly alter the HDL-LAP interaction. Therefore, additional compartments are not justified under these experimental conditions. In fact, the high correlation between the experimental data and those calculated from the model gives us added confidence in the validity of the parameters predicted by the model. These are mainly the organ distribution volumes of HDL and the in vivo partition coefficient of each LAP between HDL and the aqueous phase.

Organ distribution volumes of $H D L$. Our study quantitatively distinguishes the organ distribution volumes of HDL from those of free apopeptides. Although the degree of uncertainty of parameters calculated from a model are not easily comprehended, our data clearly indicated that HDL distribute into three organs; liver, adrenals, and ovaries. As mentioned above, we have considered HDL as a single compartment, although the heterogeneous nature of HDL is well established. The interaction of the LAPs with the different HDL subfractions is not necessarily the

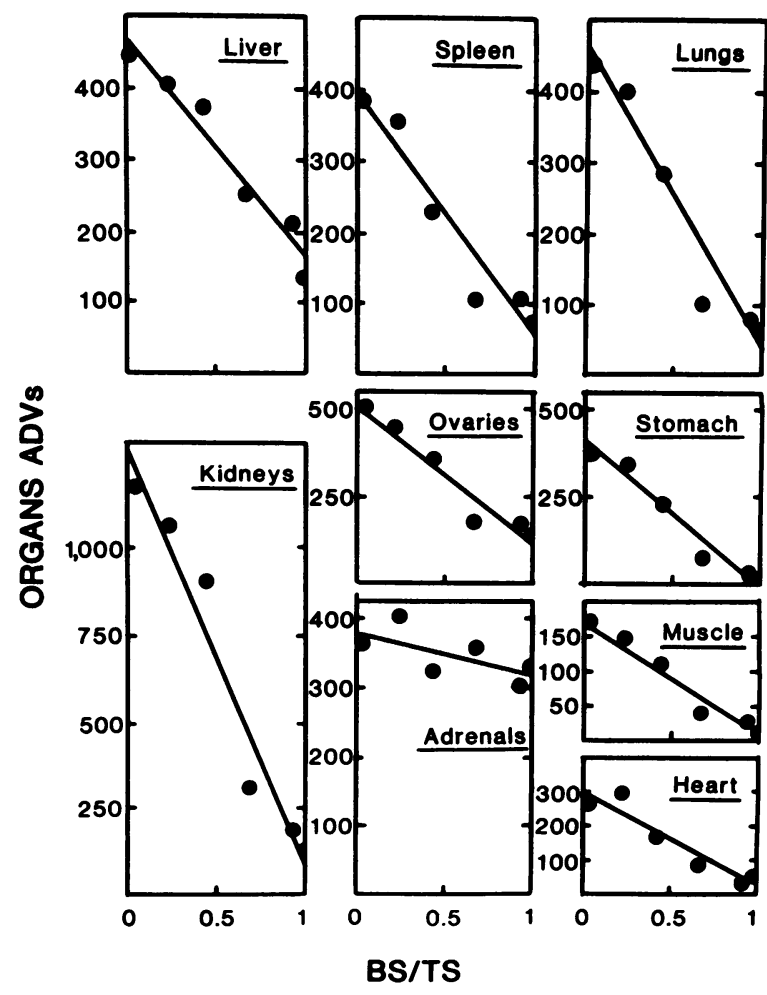

Figure 9. Final estimates of the model parameters. The organ ADVs were plotted vs. BS/TS according to equation 2 of the model described in Methods. For each organ the plot is that of a straight line whose slope is $-\Delta$ and $y$-intercept is $\mathrm{ADV}_{0}$. All the parameters are defind in the legends to Figs. 5 and 6. 


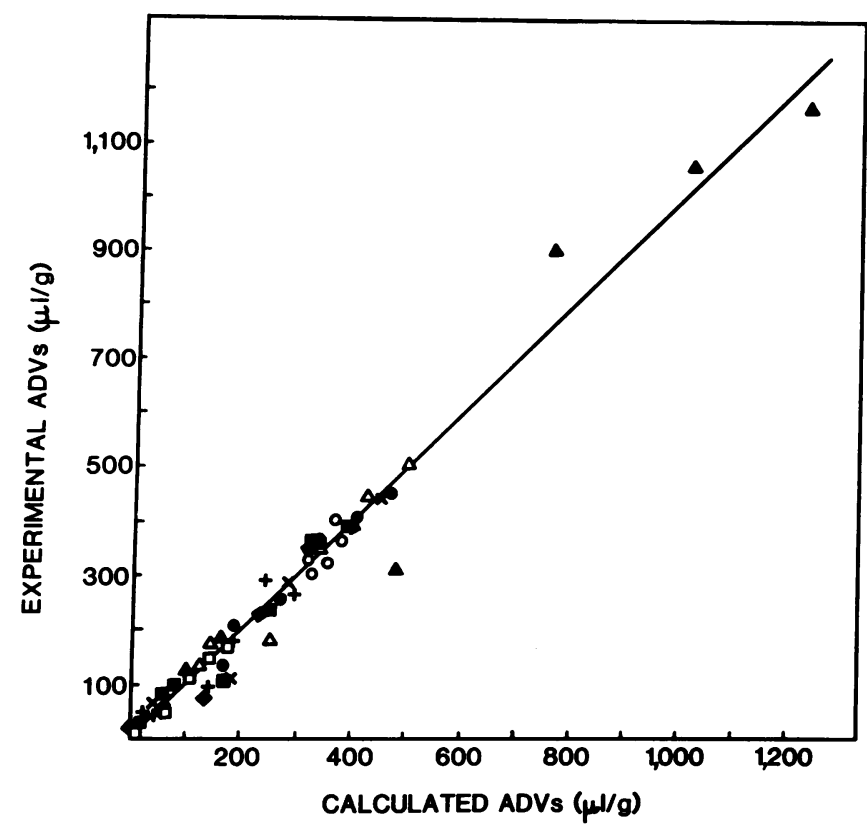

Figure 10. Correlation between the experimental distribution volumes of $\mathrm{C}_{\mathrm{n}}$-LAPs in organs and those calculated from the model described in Methods. The symbols correspond to different organs: liver, ๑; kidneys, $\Delta$; adrenals, O; ovaries, $\Delta$; spleen, $\square$; muscle, $\square$; heart, +; lungs, $\times$; and stomach, $\bullet$. The correlation coefficient was $r=0.984$.
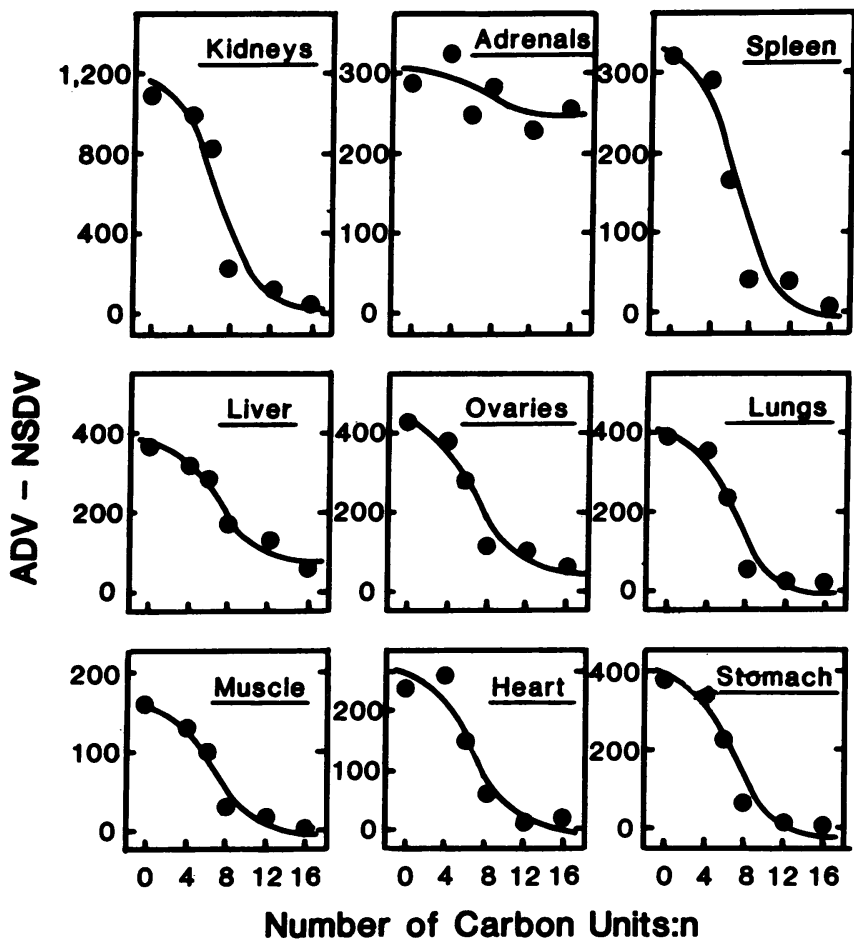

Figure 11. Relationship between the organ distribution volumes of the LAPs (ADV) and the number of carbon units of their acyl chain (n). The experimental data are shown together with the theoretical curves obtained for each organ from: $A D V-N S D V=\left(B D V e^{\wedge} e^{K n}+F D V\right) /$ $\left(\mathrm{e}^{A} \mathrm{e}^{\mathrm{Kn}}+1\right)$, where BDV and FDV are the distribution volumes in each organ of the HDL-bound-LAP and free LAP, respectively. NSDV represents the nonspecific association of the LAP in each organ due to the presence of residual serum. NSDV has been determined after injection of ${ }^{125} \mathrm{I}$-albumin. $\mathrm{A}$ and $\mathrm{K}$ are, respectively, the $y$-intercept and the slope of the straight line shown in Fig. 7.

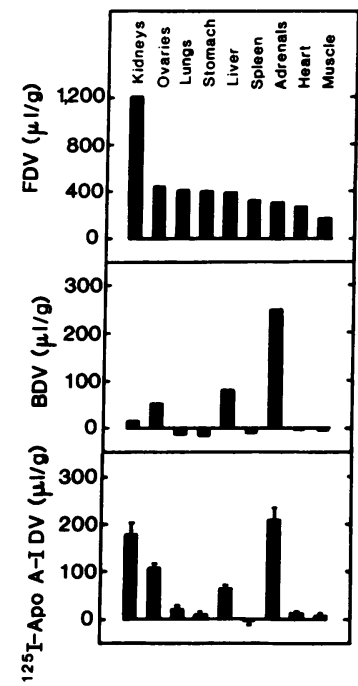

Figure 12. Distribution volumes in organs of free (FDV) and HDL-bound LAP (BDV). The values of FDV (top) and BDV (middle), expressed per gram of tissue, have been calculated from the model described in Methods. Bottom, for comparison, the experimental distribution volumes obtained after injection of rat ${ }^{125}$ I-apo A-I-labeled HDL prepared according to (11). These data have been corrected for NSDV. same as that of native HDL apoproteins. Moreover, the association of HDL with any given organ may vary from a HDL subfraction to another. Therefore, the calculated organ distribution volumes of HDL-bound LAP must be considered only as an average distribution of $\mathrm{HDL}$, in the rat. Under these conditions, the liver is the organ that binds most of the HDL, although adrenals have the highest affinity of any organ per gram of tissue. These results agree with recent reports indicating that, in rats, HDL are the main suppliers of cholesterol for the steroidogenic tissues $(26,29-30)$. Interestingly, we did not find any significant amount of HDL-bound peptide in kidneys. In contrast, kidneys were extensively labeled after injection of rat ${ }^{125} \mathrm{I}$ apo A-I, which suggests that the uptake of apo A-I by kidneys involves free rather than HDL-bound apoprotein. This finding is consistent with the hypothesis of Glass et al. (11). After studies of tissue degradation of apo A-I in the rat, these authors postulated the existence of a free apo A-I pool distinct from HDL, which was supposed to be mainly catabolized by kidneys. A major role of kidneys in the catabolism of apo A-I or apo A-II would be tenable since these apoproteins have molecular weights small enough to pass through the glomerular barrier (31). From this viewpoint, a comparison between the organ distribution volumes of apo A-I and apo A-IV would be of great interest since the latter is probably too large for significant amounts to be filtered by the kidneys.

In vivo partitioning of apolipopeptides. One of the parameters predicted by our model is the in vivo partitioning of each acylated

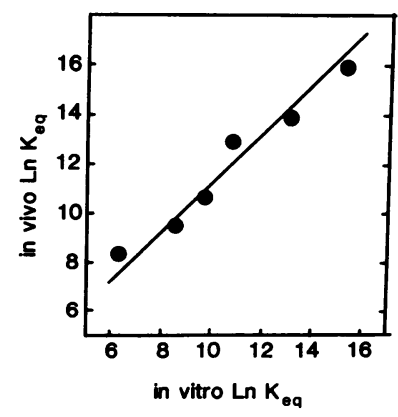

Figure 13. Correlation between the constants of equilibrium $\left(K_{\text {eq }} s\right)$ for the binding of the LAPs to HDL in vivo, with those previously obtained in vitro. $K_{\text {eq }} \mathrm{s}$ were calculated from: $K_{\mathrm{eq}}=\mathrm{BS} / \mathrm{FS} \times[\mathrm{W}] /$ $[\mathrm{P}]$, where $[\mathrm{W}]$ is the molar concentration of water $(55.5 \mathrm{M})$ and [P] is the molar concentration of HDL phospholipid. [P] was calculated from an average of literature data (28). BS/FS was calculated as described in the legend to Fig. 7.

In vitro $K_{\text {eq }} \mathrm{s}$ were those calculated in a previous work (21). 
LAP between HDL and the aqueous phase of serum. From these partition coefficients, we calculated the corresponding equilibrium constants $\left(K_{\mathrm{eq}} \mathrm{s}\right)$. These were strictly correlated with the corresponding values previously obtained in vitro (21). However, the in vivo $K_{\text {eq }} \mathrm{s}$ were approximately three times higher than the in vitro $K_{\mathrm{eq}} \mathrm{s}$. This difference is relatively small and may be explained on the following basis. First, to calculate the in vivo $K_{\text {eq }}$ s, we estimated the concentration of HDL phospholipids from an average of literature data. Since these vary greatly from one study to another, the value that we used is not necessarily perfectly representative of our particular group of rats. Second, the in vitro calculated $K_{\text {eq }}$ sere those of the binding of the LAPs to apo A-I/POPC complex, whereas the in vivo values were based upon the binding of the LAPs to native HDL. As in the case of the in vitro measurements, there was a log-linear relationship between the in vivo $K_{\text {eq }}$ s and the number of carbon units of the acyl chain of the LAP. According to previous work, this is characteristic of a hydrophobic effect $(32,33)$, and implies that the contribution of each carbon unit to the free energy of association $\left(\Delta \mathrm{G}_{\mathrm{a}}\right)$ was the same. This contribution was $-0.30 \mathrm{kcal} \mathrm{mol}^{-1}$ per carbon unit, which is virtually identical to the previously calculated in vitro value $\left(-0.35 \mathrm{kcal} \mathrm{mol}^{-1}\right.$ per carbon unit). Therefore, the binding of the LAPs to HDL in vivo presents characteristics very similar to those obtained in vitro, namely, (a) the absolute values of $K_{\text {eq }} \mathrm{s}$ are comparable, $(b)$ in both cases, there is a log-linear relationship between $K_{\mathrm{eq}} \mathrm{s}$ and the number of carbon units of the LAP acyl chain, and $(c)$ in both cases, the contribution of each carbon unit to $\Delta G_{a}$ is identical. These notable similarities clearly show that the hydrophobic properties of the LAPs are fully expressed in vivo. This illustrates the large effect of hydrophobicity on the binding of the LAPs to HDL, which went from virtually 0 with $\mathrm{C}_{0}$-LAP to $100 \%$ with $\mathrm{C}_{16}$ LAP. Finally, our work shows that a rule that we previously established in vitro applies in vivo: for a given family of apolipopeptides having a constant putative $\alpha$-helicity and a constant amphipathic length, the binding to lipid surfaces is governed by their hydrophobicity. Therefore, an important factor that regulates the partitioning and catabolism of a given apoprotein is its relative solubility in the aqueous phase of serum and in the lipid surface of its specific lipoprotein carrier. The question as to whether this rule applies in other species including humans has not been directly answered in this work. However, it should be pointed out that the hydrophobic interaction results from intrinsic physico-chemical properties of the lipoprotein-apoprotein complex. Therefore, its generalization seems reasonable, although this does not exclude the possibility of additional less conventional mechanisms of lipoprotein-apoprotein interaction resulting from a specific biological process which might occur in a given species.

Biological implications of the hydrophobic effect. Our demonstration of the role of hydrophobicity on the lipoproteinapoprotein interactions might be generalized to other systems. Indeed, several naturally occurring substances including some hormones, vitamins, or carcinogens are relatively hydrophobic. Thus, their lipid solubility might affect their metabolic fate. On the other hand, our study emphasizes the possibility of varying the hydrophobicity of certain drugs as a way of improving control of their metabolism and/or pharmacological effects. From this viewpoint our study of acylated LAPs shows that the alteration of the hydrophobic properties of a compound may have highly predictable metabolic consequences.

\section{Acknowledgments}

The authors wish to thank Sarah Myers-Fossett for preparation of the manuscript and Susan Kelly for providing the line drawings.

This research was supported by grants from the National Institutes of Health (HL-26250 and HL-27341) and the Robert A. Welch Foundation (Q-906).

\section{References}

1. Havel, R. J. 1975. Lipoprotein and lipid transport. Adv. Exp. Med. Biol. 63:37-59.

2. Eisenberg, S., and R. I. Levy. 1975. Lipoprotein metabolism. $A d v$. Lipid Res. 13:1-89.

3. Smith, L. C., H. J. Pownall, and A. M. Gotto. 1978. The plasma lipoproteins. Structure and metabolism. Ann. Rev. Biochem. 47:751777.

4. Shen, B. W., A. M. Scanu, and F. J. Kezdy. 1977. Structure of human serum lipoproteins inferred from compositional analysis. Proc. Natl. Acad. Sci. USA. 74:837-841.

5. Schaefer, E. T., S. Eisenberg, and R. I. Levy. 1978. Lipoprotein apoprotein metabolism. J. Lipid Res. 19:667-687.

6. Imaizume, K., M. Fainaru, and R. J. Havel. 1978. Composition of protein of mesenteric lymph chylomicrons in the rat and alterations produced upon exposure of chylomicrons to blood serum and serum proteins. J. Lipid Res. 19:713-722.

7. Patsch, J. R., A. M. Gotto, Jr., T. Olivecrona, and S. Eisenberg. 1978. Formation of high density lipoprotein ${ }_{2}$-like particles during lipolysis of very low density lipoproteins in vitro. Proc. Natl. Acad. Sci. USA. 75: 4519-4523.

8. Pownall, H. J., Q. Pao, M. Rohde, and A. M. Gotto, Jr. 1978. Lipoprotein-apoprotein exchange in aqueous systems: relevance to the occurrence of apo A-I and apo C-proteins in a common particle. Biochem. Biophys. Res. Commun. 85:408-414.

9. Pownall, H. J., D. Hickson, and A. M. Gotto, Jr. 1981. The free energy of association of lecithin with reduced and carboxymethylated apolipoprotein A-II from human plasma high density lipoprotein. $J$. Biol. Chem. 256:9849-9854.

10. Schonfeld, G., A. Bailey, and R. Steelman. 1978. Plasma apolipoprotein A-I and A-II levels in hyperlipidemia. Lipids. 13:951-959.

11. Glass, C. K., R. C. Pittman, G. A. Keller, and D. Steinberg. 1983. Tissue sites of degradation of apoprotein A-I in the rat. J. Biol. Chem. 258:7161-7167.

12. Segrest, J. P., R. L. Jackson, J. D. Morrisett, and A. M. Gotto, Jr. 1974. A molecular theory of lipid-protein interactions in the plasma lipoproteins. FEBS (Fed. Eur. Biochem. Soc.) Lett. 38:247-253.

13. Sparrow, J. T., A. M. Gotto, Jr., and J. D. Morrisett. 1973. Chemical synthesis and biochemical properties of peptide fragments of apolipoprotein-alanine. Proc. Natl. Acad. Sci. USA. 70:2124-2128.

14. Sigler, G. F., A. K. Soutar, L. C. Smith, A. M. Gotto, Jr., and J. T. Sparrow. 1976. The solid phase synthesis of a protein activator for lecithin-cholesterol acyltransferase corresponding to human plasma apoC-I. Proc. Natl. Acad. Sci. USA. 73:1422-1426.

15. Mao, S. J. T., J. T. Sparrow, E. B. Gilliam, A. M. Gotto, Jr., and R. L. Jackson. 1977. Mechanism of lipid-protein interaction in the plasma lipoproteins: Lipid-binding properties of synthetic fragments of apolipoprotein A-II. Biochemistry. 16:4150-4156.

16. Chen, T. C., J. T. Sparrow, A. M. Gotto, Jr., and J. D. Morrisett. 1979. Apolipoprotein A-II. Chemical synthesis and biophysical properties of three peptides corresponding to fragments in the amino-terminal half. Biochemistry. 18:1617-1622.

17. Fukushima, A., S. Yokoyama, A. J. Kroon, F. J. Kezdy, and E. T. Kaiser. 1980. Chain length function correlation of amphiphilic peptides. Synthesis and surface properties of a tetratetracontrapeptide segment of apolipoprotein A-I. J. Biol. Chem. 255:10651-10657.

18. Kanellis, P., A. Y. Romans, B. J. Johnson, H. Kercret, R. Chio- 
vetti, T. M. Allen, and J. P. Segrest. 1980. Studies of synthetic peptide analogs of the amphipathic helix. Effect of charged amino acid residue topography on lipid affinity. J. Biol. Chem. 255:11464-11472.

19. Pownall, H. J., A. Hu, A. M. Gotto, Jr., J. J. Albers, and J. T. Sparrow. 1980. Activation of lecithin:cholesterol acyltransferase by a synthetic model lipid-associating peptide. Proc. Natl. Acad. Sci. USA. 77:3154-3158.

20. Pownall, H. J., J. B. Massey, S. K. Kusserow, and A. M. Gotto, Jr. 1978. Kinetics of lipid-protein interactions. Interaction of apolipoprotein A-I from human plasma high density lipoproteins with phosphatidylcholines. Biochemistry. 17:1183-1188.

21. Ponsin, G., K. Strong, A. M. Gotto, Jr., J. T. Sparrow, and H. J. Pownall. 1984. In vitro binding of synthetic acylated lipid associating peptides to high density lipoproteins. Effect of hydrophobicity. Biochemistry. 23:5337-5343.

22. Greenwood, F. C., W. M. Hunter, and J. S. Glover. 1963. The preparation of $\left[{ }^{131} \mathrm{I}\right]$-labeled human growth hormone of high specific radioactivity. Biochem. J. 89:114-123.

23. Pownall, H. J., W. B. VanWinkle, Q. Pao, M. Rohde, and A. M. Gotto, Jr. 1982. Action of lecithin:cholesterol acyltransferase on model lipoproteins. Preparation and characterization of model nascent high density lipoproteins. Biochim. Biophys. Acta. 713:494-503.

24. Koelz, H. R., B. C. Sherrill, S. D. Turley, and J. M. Dietschy. 1982. Correlation of low and high density lipoprotein binding in vivo with rates of lipoprotein degradation in the rat. A comparison of lipoproteins of rat and human origin. J. Biol. Chem. 257:8061-8072.
25. Ponsin, G., and H. J. Pownall. 1985. Equilibrium of apoproteins between high density lipoprotein and the aqueous phase: Modeling of in vivo metabolism. J. Theor. Biol. 112:183-193.

26. Chen, Y.-D. I., F. B. Kraemer, and G. M. Reaven. 1980. Identification of specific high density lipoprotein-binding sites in rat testis and regulation of binding by human chorionic gonadotropin. J. Biol. Chem. 255:9162-9167.

27. Havel, R. J., H. A. Eder, and J. H. Bragdon. 1955. The distribution and chemical composition of ultracentrifugally separated lipoproteins in human serum. J. Clin. Invest. 34:1345-1354.

28. Chapman, J. M. 1980. Animal lipoproteins. Chemistry, structure and comparative aspects. J. Lipid Res. 21:789-853.

29. Gwynne, J. T., and B. Hess. 1980. The role of high density lipoproteins in rat adrenal cholesterol metabolism and steroidogenesis. $J$. Biol. Chem. 255:10875-10883.

30. Andersen, J. M., and J. M. Dietschy. 1981. Kinetic parameters of the lipoprotein transport systems in the adrenal gland of the rat determined in vivo. Comparison of low and high density lipoproteins of human and rat origin. J. Biol. Chem. 256:7362-7370.

31. Maack, T., V. Johnson, S. T. Kau, J. Figueiredo, and D. Sigulem. 1979. Renal filtration, transport, and metabolism of low-molecular weight protein: A review. Kidney Int. 16:251-270.

32. Tanford, C. 1980. The hydrophobic effect. Wiley, New York. 528.

33. Pownall, H. J., D. L. Hickson, and L. C. Smith. 1983. Transport of biological lipophiles. Effect of lipophile structure. J. Am. Chem. Soc. 105:2440-2445. 